\title{
GOVERNANCE, COMPLEXITY AND DEEP SYSTEM THREATS
}

\author{
Nick McDonald ${ }^{1}$ \\ Pernilla Ulfvengren ${ }^{2}$ \\ 1) CIHS, Irland \\ 2) INDEK, KTH, Sweden
}

\begin{abstract}
Aviation, health care and financial services are increasingly stretched due to aspects that pose deep enduring systemic threats to our societies, challenging our ability to respond with commensurate socio-technical solutions. It has been argued that complex systems like these are intractable, defying generalisable analysis that could support prediction and control, and hence are not amenable to compliance models of regulation. Instead it is argued here that this ability can be developed with applying governance to a knowledge system.
\end{abstract}

The knowledge system needs to identify relevant system properties with leverage on operational risk. Big data analysis plus model-based reasoning, can identify generic sociotechnical system characteristics. To make sense of the relations between system and outcome a complementary capability to model the functionality of producing the data is needed.

Our socio-technical analysis model is based on the following principles: purposive human systems have outcomes and produce value; this involves at least a minimal sequence of activity with related dependencies; it is the reciprocal nature of social relations that makes that sequence possible, and the flow of knowledge and information enables these productive roles of people. A governance system is required to assure that this works.

A governance system should generate a motivation, an "obligation to act" to use the knowledge directly within operations, to implement and validate solutions, and to manage risk across the system. This behaviour needs to be sustained in three cycles of governance:

Operational, Improvement and Strategic. The operational feedback loop maintains its role to ensure close monitoring of the operational impact of the system change, maintaining a close link between strategic implementation and operational experience.

Safety is not something distinct and separate from other aspects of system functionality, but it needs to be integrated into a new evidence-based governance of operational risk which is outlined in this paper.

Keywords: operational risk, governance, sociotechnical system analysis

(C) 2019 Authors. This is an Open Access article distributed under the terms of the Creative Commons AttributionNonCommercial 4.0 International License (http://creativecommons.org/licenses/by-nc/4.0), permitting all non-commercial use, distribution, and reproduction in any medium, provided the original work is properly cited.

ISBN: 978-91-88898-41-8

DOI: https://doi.org/10.15626/rea8.08 


\section{INTRODUCTION}

Changes in climate, demography and technology pose deep enduring systemic threats challenging our ability to respond with commensurate socio-technical solutions. For example:

In aviation, lack of technological solutions to reduce emissions means more emphasis on operational mitigation of environmental risk, finely balancing this with cost, safety and efficiency.

In financial services new technologies have transformed basic business operations such that those with corporate oversight may not fully understand their systemic operational risks. Are models of banking supervision sufficiently robust to provide regulators with accountability for the management of such risks?

In healthcare, changing demographics, mushrooming demand and escalating costs are driving constant change towards higher efficiency, while at the same time a continuing high rate of care related injuries escalates the need for assurance and management of quality and safety.

The challenge is to design whole integrated systems that perform optimally both locally and globally. Failure to address system complexity adequately will lead to partial, ineffective solutions to deep system threats and ultimately to lack of trust in critical operational institutions.

It has been argued that these complex systems are intractable, defying generalisable analysis that could support prediction and control, and hence are not amenable to compliance models of regulation. Furthermore, it is contended that contextually-grounded informal modes of selforganisation and learning appear as the only viable approach, though these provide little assurance, or evidence, of effectiveness.

On the contrary, it is argued here that when socio-technical systems function well, the ability to respond to the challenges above becomes realistic. Well-functioning systems fulfil the needs they were designed for. This implies deep, ecologically valid knowledge of how the system functions and this in turn implies an analytic capability that is proportional to the complexity of the system. This knowledge in itself is not enough - we need effective governance to ensure increasingly reliable achievable of outcomes that are planned and needed.

\subsection{Socio-technical functionality in tractable systems}

Throughout the history of production and work organisation there is research evidence and best practices showing common factors for success in tractable systems.

Total quality management (TQM) [1] is a management concept for production systems with focus on reducing variations of product quality. "Six sigma" and the focus on quantitative and statistical analysis is often associated with TQM. In Time based management (TBM)[1] the production system was managed with respect to time and an industry example (T50) shows that cutting the production time in half was doable. Lean, similarly, manages production systems with respect to a number of waste categories [1]. The different performance management foci is what discriminates these concepts from one another.

What is interesting in this context is what they have in common: the human activities that explain their success. In TQM the core idea was to mobilise people at work to join forces towards the strategic goals of the company and through local quality circles with their knowhow improve the processes. The TBM success is explained with quality circles, flatter 
organizations, decentralized decision-making, individual responsibility, broader assignments, team work, increased dialogue and democracy between managers and workers (co-workers), internal mobility and flow organization. Lean is building on the former and is making it work through values like respect for the individual, elaborate structure for meetings of daily pulse meetings and continuous improvement activities. All these human activities represent parts of a knowledge system involving the know-how from the work processes and activities central for the value creating processes. . In retrospect realisation and implementation of new production or management concepts have been successful due to common functionalities that links to human knowledge and know-how of the work they perform, managers that have understood the importance of democratic dialogue with those with know-how and across functions in the organisation due to process management and communication tools information may flow to relevant functions in the organisation. A combination of governance and self-organisation.

The production systems used in these examples are relatively stable and tractable systems and they are mostly operated in a controlled environment and there is a large amount of reproduction of work.

\subsection{Socio-technical functionality in management}

In business process re-engineering (BPR) [4] work organization changes from manufacturing was adopted for management. Functional management was transformed to process management, similar to a variation of manufacturing layouts from functional groupings of machines to a product layout. The human activities at work changed from standardized mass production lines with high work tempo to a self-organized work in teams and operators with multiple skills for redundancy. In BPR "white collar" work was similarly re-organized into teams from various departments and again, hierarchy, role and work description was making room for broader assignments managed by groups in a flatter hierarchy. Activities were no longer measured per se, now the final results counted.

Systems theory is the basis for many methods and models in work science [5]. Another field where system theory is core is in systems engineering. Although mostly intended for complex technical systems. With 'The Fifth Discipline', Senge [6] argued for the value of these systems engineering approaches to the management community. His work focuses on the social processes of engineering in support of the complexity of management processes and its structures. System thinking was facilitating communication between people at different physical places in an organization and to break departmental "silos".

\subsection{Tractability}

Why is it not possible simply to extend these ideas to solve the crises outlined above? The critical issue is the contrast between systems which, in their fundamental organization, are largely linear and often mechanically determined and systems where the fundamental control and co-ordination is done by people operating the system, where their local know-how may not be fully available outside that operational context. Hollnagel expresses the distinction in terms of tractability:

"If we do not have a clear description or specification of a system, and/or if we do not know what goes on 'inside' it, then it is clearly impossible to control it effectively, as well as to make a risk assessment. We can capture these qualities by making a distinction between tractable and intractable systems.... A system is tractable if the principles of its functioning are known, if descriptions of it are simple and with few details and, most importantly, if it does not change while it is being described. An example could be an assembly line or a suburban railway. Conversely, a system is intractable if the principles of its functioning are only partly known (or, 
in extreme cases, completely unknown), if descriptions of it are elaborate with many details and if systems change before descriptions can be completed. " [7] p.118

This creates a paradox that needs to be resolved - how to understand factors that are not generically comprehensible; and how to control factors that are inherently intractable? How to understand the relation between system and outcome. These problems are real and faced on a daily basis by those responsible for managing safety and risk in industries as diverse as aviation, healthcare and financial services. They are manifest in recurrent problems in a wide variety of areas including the following:

- The gap between a standard operating procedure and how work is actually carried out

- The ability to actually learn from safety failures by implementing preventive measures in a verifiably effective manner

- Being able to move from a reactive approach of responding to incidents after they have occurred to proactively anticipating and monitoring known risk conditions or even exploring potential risks not yet realised in a fully preventive fashion.

- Being able to design and implement new technologies and technical systems in a way that avoids operational risk.

New models of regulation (e.g. ICAO) [8] increasingly aspire to addressing these issues, but still leave gaps in prescribing how they can actually be achieved [9]. How to make that complexity tractable?

Braithwaite et al. [10][11] see the problem in terms of Complex Adaptive Systems. They counterpose 'top-down planning' with the 'self-organizing' of complex systems, but this seems to restate the problem without providing much leverage over the solution. These papers are concerned with healthcare outcomes (both positive and negative) and the complex antecedents of these outcomes. This implies some kind of complex causal model. However, to understand such complex patterns of interaction requires greater standardization of analytic frameworks than is demonstrated in the work cited; the lack of such a framework emphasises the apparent intractability of the analysis of such complex systems. The role of governance is recognized but only as a background conditioning factor, without much analysis. Thus, the capability to govern productively in a way that maximises success is not seen to be a strategic objective. Furthermore, it is not recognized that good governance may be a precondition for gathering evidence about implementation and change. Without it there may be no basis for good practice or learning. This is not to deny the role of self-organising but to assert that good governance could facilitate self-organising.

What we are describing may fulfill the conditions for a perfect storm. Increasing system interdependency and integration potentially leads to deepening system crises if we cannot understand or control those deep system interactions. At the same time our ability to understand people and technology in systemic interaction is, if anything, decreasing in proportion to the scale of the problems that need to be understood. Hence our ability to intervene productively to ameliorate those crises decreases as the problems escalate.

The word 'tractable', meaning easily managed or controlled, perhaps disguises the problem. We need to think more in terms of 'leverage' - the exertion of power or force through the mechanism of a lever to obtain a new result. If systems are intractable (difficult to control), then we need to understand them in a way that gives access to the mechanisms that give leverage within those system relations to influence the reliability of the system output. 


\section{COMPLEXITY}

The notion of complexity of socio-technical systems seems to be at the core of this dilemma. The problem of complexity can be expressed as follows [12]. There are large numbers of elements and interactions; within these interactions each outcome can have multiple causes, but each cause can have multiple effects; these interactions can be non-linear, giving rise to great unpredictability in outcome unless one understands the system parameters (e.g. a change in the state of the system) which transform the nature of the relationship. Such systems have 'emergent properties' which are explicable in terms of the relationships between elements in the system but not in terms of the qualities of these elements themselves (nondecomposability).

The role of the human participant is crucial but brings the added complication of multiple divergent points of view, but also the possibility of self-organisation (relatively spontaneous and independent of formal organizational arrangements, as well as through established social structures). Thus, if the natural order of things tends towards disorder and chaos, then the possibility is for social systems to adapt to changing environmental demand. It is arguable that socio-technical systems are safe, effective, efficient precisely because of this capacity for self-organisation, for no amount of procedure or automation can adequately determine the human role. How then are we to understand the nature of organising activity in purposeful productive systems? How to reconcile the role of 'self-organising' with the role of governance?

The first set of complexity issues requires a new approach to knowledge creation and can be addressed by better matching the scale and quantity of data to be analysed to the nature of the system, together with explanatory system models that relate to socio-technical system functioning. This is not to suggest that such models and data can explain all such complex interactions, but merely to state that this approach should increase the power of the explanation over what is possible at present.

The second set of issues invites us to engage with the human capacity to understand, act and self-organise to develop new modes of system governance that can deliver more reliable outcomes in a transparent and accountable way. We will deal with each of these in turn.

\section{SOCIO-TECHNICAL ANALYSIS AND MODELS FOR COMPLEX SYSTEMS}

\subsection{Multiplicity of data, events, cases}

The principle of requisite scale and variety of data applies at all stages of activity: responding to events, understanding the normal operation, understanding implementation and change. Generating data and analyses can initiate a flow and feedback of resulting knowledge into the operation and into management of the system.

The basic reactive model of safety centres on responding to individual events or incidents. However, any one event cannot be representative of a complex dynamic operational system, because it is not possible to distinguish what is typical for the system from what may be idiosyncratic to that situation. It is only by pooling the results of many thorough investigations that general trends can become apparent and the problems of complexity can progressively be addressed. The quality of investigations is very important; even though single event investigations normally cannot produce strong recommendations, combined 
analysis of multiple investigations can overcome this limitation. Low quality investigations will simply mean that the complexity becomes uninterpretable.

It is also important to complement analyses of incidents with better understanding of the normal variance of operations. The rapidly escalating availability of large amounts of data from technology-supported operations has made it possible to look at relatively minor deviations in a reactive way, but increasingly to analyse large integrated databases to identify complex risk patterns with predictive analytics [13][14]. This in turn opens the possibility of linking factors proximal to the operation to characteristics of core resource inputs to the operation, for example profiles of experience, staff roster patterns, or equipment maintenance history, thus building a more complex picture of leading and lagging indicators that offers more leverage to improve the operation. This more complex picture in turn makes possible a smarter feedback of risk information that is more tailored to the particular circumstances of the operation, supporting local proactive risk management. It also opens the possibility of performing operational audits that are calibrated against situationally specific operational risk, rather than simply calibrated against procedural compliance. The building of a knowledge base of system risk should also improve the quality of investigations. Event analysis is thus not an isolated bounded activity. It is part of an extended process of acquiring knowledge about the system. While the default assumption tends to be that incident management is a more or less linear process, leading from event to investigate to recommend to implement, this cannot (and does not) work.

The combination of evidence from multiple investigations, operational data analyses, audits, etc. requires a more complex organisational process than most organisations currently provide. However building this evidence base is critical for a credible plan for improvement. Moving from analysing a problem to constructing a credible solution creates a shift from identifying all the factors that may be relevant to understanding those factors that give leverage to change the system within its situational constraints. In turn the processes of planning and implementing change confront that particular solution with a whole range of potentially disruptive influences, including the normal requirements of maintaining operations and competition from other parallel initiatives and projects. All of this brings pressure not only on the quality and robustness of the original analysis, but also on the process of change itself. This brings into focus a new level of analysis of multiple change initiatives, again addressing complexity with multiplicity, in order to analyse the risk in change. Each implementation project has its own particular characteristics and circumstances and it is not clear what factors will lead to successful implementation (or otherwise). This can only be addressed by building analyses of multiple projects, over time and across large organisations, using a common methodology consistently to analyse the role and influence of those relevant factors.

The aggregation of evidence from multiple projects and their interactions can bring the management of operational risk up to strategic level in the organisation for the first time. In most organisations strategic risk and performance management tend to be financially dominated. However with an active management of operational risk at a system level it becomes possible to examine in a balanced way the overall contribution of different risks to system performance - for example, safety, cost, quality, or environmental impact. An integrated risk and performance management system would then combine technical, operational and strategic risks in a common framework. This is critical to support effective decision making - see the Governance framework below. 


\subsection{Analysing complex socio-technical systems}

Analysing large amounts of data, both quantitative and qualitative, requires a complementary capability to model the system that is generating the data in order to make sense of the relations between system and outcome. Unfortunately, such a modelling capability is not easily derivable from dominant theoretical perspectives. The following crude generalisations about different theoretical approaches and the type of knowledge they sustain exposes a critical gap in understanding the relationship between the characteristics of a socio-technical system and the quality of the productive outcomes that it sustains.

- Organisational theory tends to eschew any serious discussion of process and value creation

- Theories of business processes and value chains are weak in analysing the role of people, both individual and collective

- Theories of system improvement, like total quality management, have a strong basis in the role of people, for example in quality circles, but this has not led to a substantial theory of organising.

- Human Factors, while aspiring to be a systemic science, is much more comfortable in dealing with local interactions between people and with technologies.

- More global approaches to analysing social relations are weak on analysing the functional process in which people engage.

- Theories of information and knowledge tend to focus on the formal structural transformations of information and knowledge rather than the content of the knowledge, and hence its practical application.

If there is a gap in the theoretical framework then certain questions cannot even be asked, let alone answered. Most often the gap in the conceptual framework is not even noticed. In order to create a way forward, a new enquiry framework, the Socio-Technical Analysis Cube was created to analyse such systems and the changes they are subject to [15][16][17].

Organisational change is addressed in terms of deliberate change designed to achieve certain objectives, but invites consideration of a variety of factors which may play a role in how such change does (or does not) come about. Implementation and change have a dual function: they are a core problem to be solved; but the process of implementation is also a key source of evidence of the effectiveness of a solution.

The analysis is based on the following principles: purposive human systems have outcomes and produce value; this involves at least a minimal sequence of activity with related dependencies; it is the reciprocal nature of social relations, both in working with and reporting to others, that makes that sequence possible, and the flow of knowledge and information enables these productive roles of people. Each of these four dimensions of organisation can be described in four different ways: as a functional system; as represented (however imperfectly) by measurable data; as understood and made sense of by the people involved; and finally as collective values, norms, sub-cultures and meanings which make up the culture. Across these multiple dimensions, it is not so much the absolute values that are important but the relationship between different elements that provide a mechanism for delivering particular outcomes under certain contextual conditions. The default implementation sequence addresses the qualities of each stage: the cogency and importance of the problem diagnosis; the leverage offered by the solution; the effectiveness of the plan in reconciling conflicting requirements; the sustainability of the key elements of the solution through the implementation process; and the verifiable link of the outcome to the initial problem. This does not imply a simple linear sequence - for example, each 'stage' can (and should) involve a recombination and reorganisation of many elements; 'progress' though the stages can be halting, iterative, 
disjointed at different levels, etc. However the objective is to track whatever link there is between a recognised problem state ('risk') and an eventual outcome which mitigates this (or not).

This analytic capability underlies the building of socio-technical models. The model of Governance outlined in this paper is one such model. Other generic models of different process types have also been proposed. The Governance model addresses how the information transformed into knowledge by the analytic framework can be brought to bear on the specific human side of complexity: the complicated (difficult) problem of how to engage with human understanding and capacity for self-organisation in order to move a problem more reliably towards an effective implemented solution.

\section{GOVERNANCE}

In order to understand and project how flows of information within an organization could foster not only enhanced collective awareness but also more effective management, a model of mindful governance of operational risk was developed and, in part, validated through two industrial case studies [18][19].

Obligation to act is a behavioural-economic concept which addresses the motivation for action in a purposeful organisational system. Basically, if an issue is important, if there is a credible solution and if someone takes responsibility for acting on it, at each stage of an implementation process, then there is an increased chance of the ultimate solution to the problem being achieved. 'Obligation to Act' is made possible by the generation of leverage (potential for change) from the analysis of a system, as indicated above. It also draws on transparent accountability for action and its consequences, distributed among those with authority to act across the system; this implies a reciprocal relationship of providing support for the actions for which accountability is required. In terms of a risk-informed system, this could take the following form: 'if I give you information about risk, then you tell me how it was managed'. This creates transparency about context, action and consequence. This form of accountability contrasts with accountability meaning liability (or blame) in the case of failure. Such accountability needs to stretch horizontally as far as operational risk transcends organizational boundaries and vertically from front line operational staff up to the relevant authority. These three governance system characteristics come together to foster an 'Obligation to Act' in the following way:

- Importance: The issue needs to be seen as systemically important so as to justify action and, in principle, be tractable (allow leverage) across any relevant horizontal boundaries.

- Efficacy: The solution needs to be both practicable and cost-effective (leverage) and, in principle, there need to be available pathways towards implementing the solution (distributed authority and accountability).

- Accountability: requires that there should be an actual handover of responsibility for the next stage of activity towards a solution, with full confidence in the capability to act effectively (this requires sufficient horizontal and vertical integration to support that activity sequence, plus an effective concept of 'holding-to-account', based on real knowledge of context-action-consequence). 


\section{THREE CYCLES OF ORGANISATIONAL GOVERNANCE}

Obligation to act motivates three cycles of information flow, each of which take the form gather information, then act on that information.

\subsection{Operational cycle}

The first, at operational level, involves gathering operational information in the form of reports, investigations, audits, operational data and feeding that back into the operation. Operational reports can include both standard mandatory reports as well as enhanced voluntary reporting of any operational information worth sharing. Accessing and integrating different strands of operational data can be slow and difficult for many organisations (data exists in well-protected silos) - but once the principle is accepted and data protections agreed, the power of the analysis of large volumes of data then becomes apparent.

Integrating this diverse information in a common risk profile for the operation then sets up a channel of tailored feedback to the operation. Short term 'live' planning can mitigate some systemic risks (e.g. resource allocation to particular operations). Operational staff check in and prepare and can be updated as appropriate. Wider management functions - risk-calibrated operational audits, training focused and evaluated according to effectively addressing risk can be developed. This is where the benefits become apparent - improved, more focused, operations, increased confidence in an agile responsive system. Feedback is generated from the operation on how risks were managed.

\subsection{Improvement cycle}

The Improvement Cycle builds on the operational risk knowledge base to develop and implement improvement initiatives through projects. Aggregating multiple reports and data analyses permits a meta-analysis of common factors that transcend particular circumstances. It establishes projects with confidence that one is identifying an underlying causal pattern justifying specific improvement initiatives to break that pattern. It sets clear criteria and targets for improvement. This enables a new channel of accountability to strategic level. Development work may need to be done to fully meet operational requirements; then planning for implementation. Ultimately, projects transfer into business processes. Often, this is the most demanding phase for those managing the project, stretching their competence in unfamiliar ways. Implementation needs to be monitored (how was it done) and lead to verification of the outcome. Initial implementation should lead to full embedding in normal practice, sustained by the system as a whole. Thus, the overall impact may take some time to establish but should engender reduced frustration at recurrent problems (as they get solved) [20]. The operational feedback loop maintains its role to ensure close monitoring of the operational impact of the change

\subsection{Strategic cycle}

The strategic cycle initiates another level of knowledge integration leading to an enhanced capacity to act strategically, informed by a detailed knowledge of operational readiness to meet strategic threats. Further meta-analysis examines the interactions between different improvement projects and between those projects and business as usual in the relevant business units. This provides an integrated system risk profile. It also creates a proactive role for a Chief Risk Officer, no longer simply monitoring the risk registers of others, but actively managing complex risks across the business. This strategic risk profile will suggest key actions that need to be taken to ensure the operation is ready for any relevant foreseen threats. This provides an evidential link between operational and strategic risk management processes. 
The accumulated evidence base extends the relevant knowledge that can be brought to bear in the design of new systems (new processes, new technologies) to meet stringent operational standards. Implementation is guided by evidence-based best practice principles. The key objective is to avoid the escalating cost of failure which is so prevalent in such initiatives (for example, the cost of the new system which does not work as intended, the cost of disruption to production, the cost of recovery with new improved system, the loss of credibility and opportunity, the cost to third parties). Again, the operational feedback loop maintains its role to ensure close monitoring of the operational impact of the system change, maintaining a close link between strategic implementation and operational experience.

\section{CONCLUSION}

In summary, three emerging capabilities can combine to transform management of complex systems. New large data streams can support the analysis of inputs, activity and outputs across extended operational systems. Qualitative analytic methods that model core socio-technical dimensions during normal operations, change, crisis or future automation can complement these analyses in implementation case studies. These, in turn, enable new productive governance concepts that support appropriate and accountable action at all levels of the system. Effective governance is essential to build evidence, to enable learning and to guide future practice.

The challenge is to build a virtuous cycle: a combination of data rich analysis and modelling leading to a strong programme of implementation; implementation leading to a further flow of data and analysis from multiple cases; the whole leading to a body of increasingly sound evidence about system functioning at different levels - the core operational system, the processes of implementation and change, and the processes of governance themselves. This in turn makes possible evidence-based governance of risk within the organisation, and, in so far as processed knowledge is much more sharable than raw data, across an industry, and even between industries where lessons can be learnt.

Is it a powerful enough model to play a role in addressing deep system threats, based on the production of evidence? Only time will tell, based on the implementation of such a model. However, if this kind of model is not sufficient, then it should open the way for a more powerful model, and the evidence from failure should help design a more robust and effective model of Governance of Socio-Technical Systems. This is important because failures of governance imply failures to meet strategic objectives and inability to tackle strategic challenges. Such failure breeds mistrust of the system, which can exacerbate the problem, intensifying the perfect storm. Good governance can build trust, based on evidence that progress can be made in ameliorating strategic crises.

Hollnagel has commented: "It may also happen that the very concept of safety is gradually dissolved, at least in the way that it is used currently, as something distinctively different from, e.g., quality, productivity, efficiency, etc. If that happens - and several signs seem to indicate that it will - then the result will not be a Safety- III but rather a whole new concept or synthesis (...). So while Safety-II by no means should be seen as the end of the road in the efforts to ensure that socio-technical habitats function as we need them to, it may well be the end of the road of safety as a concept in its own right." [7] p.178

Safety is not something distinct and separate from other aspects of system functionality. Safety is an aspect of the outcome of a system that needs to be managed. As such it needs to be managed in an integrated systemic way. Safety II has not spawned a practical programme of implementation and change. Maybe it was not designed to do this, rather it was focused on 
promoting a change in mindset. Through this, it has helped to define a problem that needs to be solved. It is unlikely that the concept of safety will be gradually dissolved - but it needs to be integrated into a new evidence-based governance of operational risk.

\section{REFERENCES}

[1] Deming, W.E. (1982) Out of the Crises. Cambridge, USA: MIT Press

[2] Stalk, G. Jr. and Hout, T.M. (1990) Competing against time - How Time-Based

Competition is Reshaping Global Markets. New York: The free Press.

[3] Womack, J.P., Jones, D.T. and Ross, D. (1991) The Machine That Changed The World, New York: Macmillan Publishing Company

[4] Hammer, M. and Champy, J. (1993) Reengeingeering The Corporation, New York; HarperCollins.

[5] Meister, D. (1991) The History of Human Factors and Ergonomics, Lawrence Erlbaum Associates, Inc. New Jersey

[6] Senge, P. (1990). Fifth Discipline: The Art and Practice of the Learning Organisation, Century, London.

[7] Hollnagel, E., (2014): Safety-I and Safety-II. Farnham: Ashgate

[8] ICAO, (2018): Safety Management Manual $4^{\text {th }}$. Edition. Montreal:ICAO

[9] Ulfvengren, P., Corrigan, S. (2015) Development and Implementation of a Safety

Management System in a Lean Airline, Cognition, Technology \& Work:Volume 17, Issue 2 (2015), Page 219-236, Https://doi.org/10.1007/s10111-014-0297-8

[10] Braithwaite J., Churruca, K., Long, J.C., Ellis, L.A. and Herkes, J. (2018): When complexity science meets implementation science: a theoretical and empirical analysis of systems change. BMC Medicine (2018) 16:63 https://doi.org/10.1186/s12916-018-1057-z

[11] Braithwaite, J. (2018): Changing how we think about healthcare improvement. BMJ (2018) 361:k2014, https://doi.org/10.1136/bmj.k2014

[12]C. Mesjasz: (2010): Complexity of Social Systems. Acta Physica Polonica A Vol. 117

(4) 706-715

[13] Baranzini, D (2018). "Features of Unstable Approach in Aviation: Big Data 2.0 evidence 2018" April 2018 https://doi.org/10.13140/RG.2.2.34382.15686

[14] Baranzini, D., and Zanin, M. (2015). Risk Prediction \& Risk Intelligence in Aviation the next generation of aviation risk concepts from PROSPERO FP7 Project. ESREL 2015 25th European Safety and Reliability Conference.

[15] McDonald, N. (2018a); Introduction to the STA Cube. Working document. Centre for Innovative Human Systems, Trinity College Dublin, Ireland.

[16] McDonald, N. (2018b): The Cube and Change. Working document. Centre for Innovative Human Systems, Trinity College Dublin, Ireland.

[17] Corrigan, S., Kay, A., O’Byrne, K. Slattery, D., Sheehan, S., McDonald, N., Smyth, D., Mealy, K. and Cromie, S. (2018): A Socio-Technical Exploration for Reducing \& Mitigating the Risk of Retained Foreign Objects. International Journal of Environmental Research and Public Health 2018 Apr 10;15(4). pii: E714. https://doi.org/10.3390/ijerph15040714 [18] Callari, T. C., McDonald, N., Kirwan, B., \& Cartmale, K. (2019). Investigating and operationalising the mindful organising construct in an Air Traffic Control organisation. Safety Science (Special Issue - Mindful Organising).

[19] McDonald, N., Callari, T. C., Baranzini, D., \& Mattei, F. (2019). A mindful organising governance model for ultra-safe organisations. Safety Science (Special Issue - Mindful Organising).

[20] Ward, M., McDonald, N., Morrison, R., Gaynor, D., Nugent, T. (2010): A performance improvement case study in aircraft maintenance and its implications for hazard identification. Ergonomics 53 (2), 247-267 https://doi.org/10.1080/00140130903194138 